\title{
Estimation of Cloud Cover using Colour and Texture
}

\author{
K. Richards and G.D. Sullivan \\ Intelligent Systems Group, \\ Department of Computer Science \\ University of Reading, RG6 2AY. \\ K.Richards@reading.ac.uk.
}

\begin{abstract}
We describe methods for using colour and texture to discriminate cloud and sky in images captured using a ground based colour camera. Neither method alone has proved sufficient to distinguish between different types of cloud, and between cloud and sky in general. Classification can be improved by combining the features using a Bayesian scheme.
\end{abstract}

\section{Introduction}

In order to provide accurate local forecasts, meteorologists require good quality observations, including those of cloud. The increased use of automatic stations means that valuable visual assessment of weather conditions is lost. This paper considers colour and texture as possible features to provide automatic ground-based estimation of cloud cover.

Existing techniques for interpreting cloud images have concentrated largely on satellite images. Work by Parikh and Rosenfeld used images from both the infra-red and visible regions of the spectrum [1]. Their system extracted spectral and textural information, and reached a classification using a complex selection procedure. Other papers in this field have used these features independently [2,3].

Ground-based assessment of cloud cover provides a much finer resolution in both space and time than is possible by satellite, and can provide valuable information concerning cloud at different heights. Coomes and Harrison developed a cloud estimation system using ground-based radiometric measurements [4], but although this method provides good estimates of total cover, it was unable to distinguish between different classes of cloud.

In this paper we report experiments with colour and texture analysis on cloud images obtained from a ground-based colour camera, and demonstrate that although each provides good discrimination neither is sufficient for complete analysis. A combination of these features using a simple Bayesian scheme provides improved classification.

\section{Colour processing using normalised colour space}

Colour video cameras provide an inexpensive and easy means for capturing colour images, although the stability of the colour signal is uncertain. We captured a sample set of images under varying conditions of brightness, magnification and weather in order to 


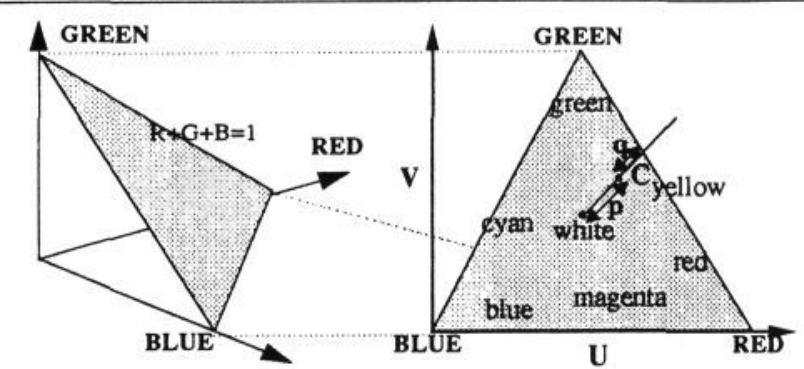

Saturation is the spectral purity of the colour.

$$
\text { Satn }=\frac{p}{p+q}
$$

Hue is quoted as the spectral colour of the vector.

Figure 1: Colour space normalisation.

find (i) what consistency can be expected from such cameras under different viewing conditions, and (ii) the separation of cloud and sky in colour space.

Early work compared a number of colour metrics and found little difference in their discriminatory abilities; consequently we have adopted a simple colour normalisation based on the CIE colour transform [5]. Each channel is normalised with respect to the total signal from all three channels, effectively projecting RGB colour space from the origin onto the plane $R+G+B=1$ (see Figure 1).

\subsection{Training set distributions}

A total of thirteen images were used for the colour training set and covered a range of weather conditions, from overcast to very sunny. For each image, masks were manually created to identify regions as cloud or sky. This was critical to our analysis since mislabelling would introduce errors into the final colour distributions, consequently classification was conservative and ambiguous regions were excluded from further analysis.

The pixels within each classified region were used to generate colour space distributions for cloud and sky. On an image-by-image basis it was evident that cloud and sky were distinct. To examine the class distributions, the results for single images were combined. Three dimensional plots of the class distributions are shown in Figure 2.

Clearly there is some overlap between sky and cloud, and to estimate the extent of overlap a confusion matrix was generated (Table in Figure 2). From these distributions the confusion between cloud and sky appears to be less than $3 \%$, therefore the CCD colour camera is sufficiently stable to offer good distinction between cloud and sky over the range of conditions in this sample set.

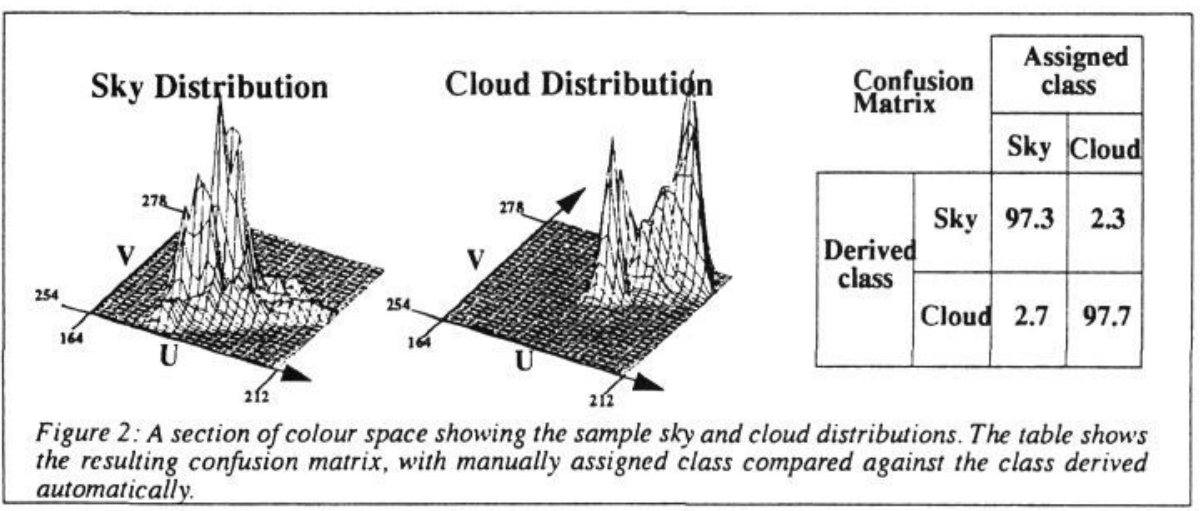




\subsection{Classification using colour}

A further set of images was captured under a wider range of conditions than with the original training set. These were classified pixel-by-pixel by looking up the most probable classification based upon the sample distributions. Typical results are shown in Figure 3, where the relative probability of cloud is shown as intensity.
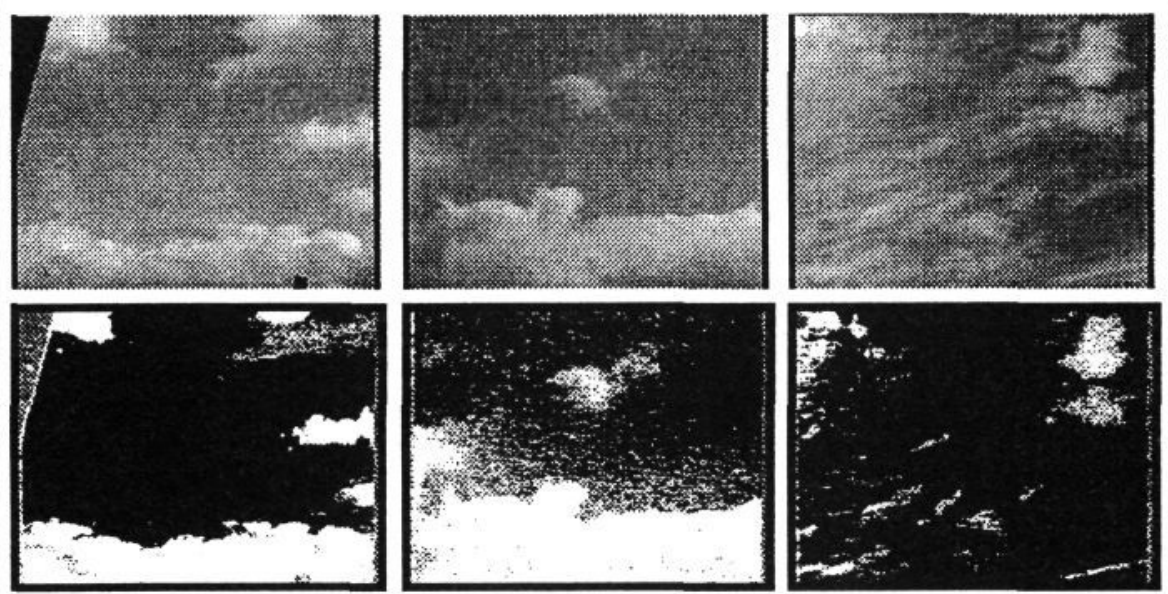

Figure 3: Colour Segmentation of cloud images. In the lower images the intensity indicates the relative probability of cloud against sky (white $=$ cloud, black $=s k y$ )

The left image shows a clear sky bordered by several cumulus clouds of varying size. The top right corner of this image contains a shadowed base of some cumulus surrounded by a small amount of haze. For the purposes of this report, haze is defined as whitish sky arising from illumination of pollution or other particles in the atmosphere. The results of segmentation are clearly good; all the cumulus clouds are located including the shadowed section, although there is some confusion shown by the grey areas (top right of left image in Figure 3).

The image in the middle column was taken with a telephoto lens and shows a border of cumulus at the bottom, with a fragment of cumulus slightly above, and a small degree of haze near the borders. There also appears to be some surrounding fibrous elements of cloud. This classification highlights the problem of haze in colour analysis. Chromatically it is very similar to cloud and results in the mis-classification of sky.

The right image contains a large amount of cirrus, which appears wispy and transparent. The image also contains obvious cumulus in the foreground, to the right and left comers. Again this image demonstrates a problem with colour segmentation; thin cloud such as cirrus appears bluish. This leads to an obvious colour ambiguity between such cloud and clear sky.

Nevertheless we have found that inexpensive CCD technology can provide fairly stable colour images, and for the purpose of cloud segmentation a simple colour metric works well. The approach is largely unaffected by changes in brightness, but several difficulties remain: (i) overestimation of cloud due to surrounding haze (see middle Figure 3), and (ii) highly textured cloud such as cirrus is missed (see right Figure 3). 


\section{Texture analysis of cloud images}

Texture has been successfully employed to classify satellite images of cloud, and we have seen that on the basis of intensity and colour there is little difference between transparent cloud (such as cirrus) and sky. Texture may provide a means to distinguish between them, and may also be useful for recognising different classes of cloud such as cumulus, stratus and cirrus.

\subsection{Laws' texture description}

We have considered a number of approaches including second order statistical moments and fractal texture models [8,9]. A texture model which has received considerable interest over recent years is based on local linear transforms. We have found this approach provides much better classification than with either of the other two approaches, although it does result in an $\mathrm{n}$-fold increase in computation (where $\mathrm{n}$ is the number of local linear transforms).

The image is first transformed using 9 local linear operators which were defined by Laws [6]. These make use of a number of empirical linear transformations which are intended to extract elementary underlying structures such flats, spots and edges. For any given point an energy measure, such as the variance or some higher order statistical measure, is calculated over a local neighbourhood in each of the transformed images. The local texture properties of that point are described by the vector of feature energy measures. Figure 4 shows Laws' spatial-statistical texture model diagrammatically.

Unser extends this work, attempting to provide a more formal basis for such texture analysis [7]. He demonstrates how optimal transformation filters may be calculated for two textural classes and also proposes sub-optimal transforms, such as the Discrete Sine Transform (DST) and the Discrete Cosine Transform (DCT), which provide good discrimination over a broad range of textures.

For the purposes of comparison we applied both Laws' $3 \times 3$ linear transforms and Unser's sub-optimal DST to the data. The DST performed very well on a number of images, but Laws' transforms consistently produced more accurate classification.

\subsection{Bayesian classification using feature vectors}

Assuming the class conditional probability distributions to be multivariate gaussian distributions, Unser uses a Bayesian classifier which minimises the possibility of misclassification. Given a set of class-mean vectors $v$, and their covariance matrices C, a
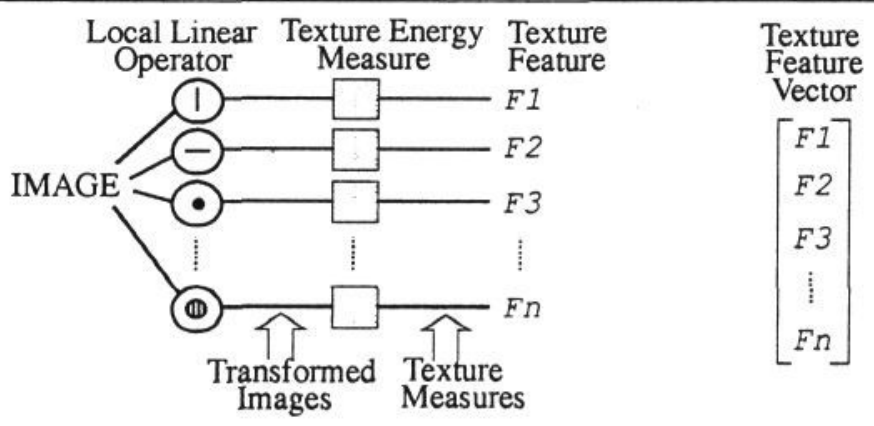

Figure 4: Laws' spatial-statistical texture model. 
feature vector $f$ can be classified by calculating the distance to each of the class-mean vectors and selecting the closest. Using a Bayesian classifier, the distance measure reduces to a simple matrix multiplication [7].

$$
d_{i}=\left(\left(f-r_{i}\right)^{\dot{T}} \bullet C_{i}^{-1} \bullet\left(f-r_{i}\right)\right)+\log \left\{\mid C_{i}\right\}
$$

$[\mathrm{i}=1, \ldots, \ldots, \mathrm{n}]$

The original colour training set was expanded to include a much wider range of cloud types, providing a number of texture samples for cirrus, haze, and cumulus. A total of twenty-eight images were used for this second training set. The class-mean vectors and covariance matrices were calculated using Laws' $3 \times 3$ linear transforms followed by a moving $8 \times 8$ window to calculate the energy measure. Classification used the same process, with the Bayesian classifier labelling each point with the nearest class (either sky, haze, cirrus or cumulus).

\subsection{Classification using Laws' texture measures}

The images classified earlier using colour were reclassified using Laws' texture measure (see Figure 5). The black regions indicate the class sky, the dark grey regions represent haze, light grey regions represent cirrus, and the white regions are cumulus.

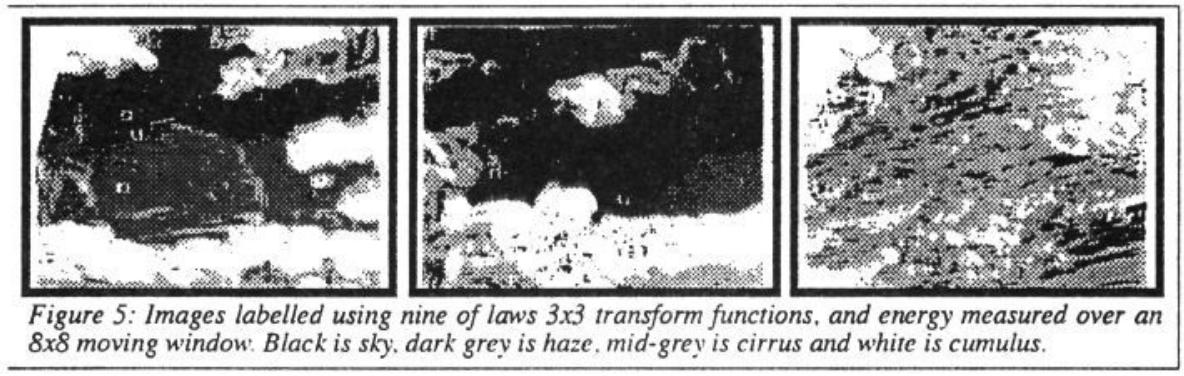

The left image seems to be quite accurately classified: the central region of sky has now been divided into two regions of sky and haze. with evidence of some small regions of cirrus. The major bodies of cumulus seem to be labelled accurately, although small parts of cumulus are mis-classified (see bottom and top right section of the image in Figure 5). These are very smooth areas of cloud and are consequently very difficult to distinguish from sky using texture.

The middle image is encouraging since it shows correctly labelled magnified regions of cloud. The majority of cloud is cumulus (with small wisps of cirrus and haze in the surrounding sky). The successful classification of magnified cumulus using texture is somewhat surprising, but may possibly be due to a fractal nature of such cloud. However, such a discussion is beyond the scope of this paper.

Finally, with the right image we can see that the image containing a large quantity of high cirrus is now classified fairly well, although some of the cirrus is mislabelled as cumulus.

In conclusion, texture is useful, but there are two difficulties with the approach: (i) sky has no texture, so that the borders of cloud extend into regions of sky causing a small overestimation of cloud; (ii) some regions of cloud have little texture and are indistinguishable from sky, they are therefore consistently mislabelled (see regions of cumulus in left and middle image of Figure 5). 


\section{Using colour and texture}

Both colour and texture have been shown to be important indicators to the presence of cloud, but neither is sufficient for complete segmentation. Chromatically, haze is indistinguishable from cloud, and cirrus is indistinguishable from sky. Texturally, smooth regions of cloud are frequently mis-labelled as sky. There is a clear attraction in using a combination of both methods.

\subsection{Combining colour and texture}

Normalised colour space produces two chromaticity measures. As we have already discussed texture can be described by a vector of spatio-statistical measures. By creating a combined feature vector using colour metrics and texture features, a description can be made in terms of both colour and texture. As with the texture classification a Bayesian classifier can be employed to minimise the probability of mis-classification.

The second training set of images was reprocessed to calculate the new class-mean feature vectors consisting of texture and colour measures. The classification procedure was based on that used for texture analysis. The results of classification using the combined colour and texture approach can be seen in Figure 5.
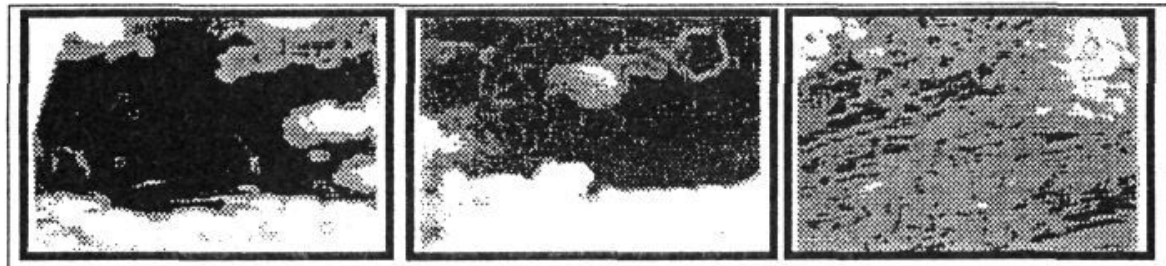

Figure 6: Images labelled on the basis of chromaticity and texture features.

\subsection{Classification using colour and texture}

The segmentation of the left image again seems good; the regions of haze located using texture are now correctly labelled as sky. The kind of improvement possible from using both texture and colour is demonstrated with the right image. Even using texture classification a number of cumulus patches were mistakenly found within cirrus. Using colour and texture these have been reduced considerably, the few remaining small patches most probably do belong to the lower cumulus clouds.

The centre image in Figure 5 is still troubled by noise, which is surprising since texture processing produced very clear regions of sky. It is difficult to determine whether this noise is an image artifact from magnification or an accurate interpretation of the data. At this degree of magnification small elements of cloud are likely to exist near cloud borders. This difficulty highlights one of the problems with interpreting cloud images, both by Man and machine.

This combination of colour and texture can greatly improve classification but is still imperfect. Obvious errors which persist from texture classification are the misclassification of smooth regions of cloud (see the top right of first image in Figure 5), and the extended classification of cloud at borders with sky. 


\section{Conclusion and future work}

It has been demonstrated that both colour and texture are useful features in the differentiation of cloud from sky, although neither approach has proved sufficient for complete analysis. A simple method to combine the features using a Bayesian classifier has shown an improvement in classification over the two independent approaches, but there are still problems to be resolved concerning: (i) cloud border accuracy, and (ii) the weighting of the classifier towards texture.

The approach presented here is suitable for simple models, but part of the success relies on the feature distributions being normal. This cannot be guaranteed in a problem domain such as this, since the radiation passing through the atmosphere is known to change throughout the day. In order to compensate for this, and deal with extreme situations such as sun-rise and sun-set, a more elaborate technique for combining colour and texture is required.

Despite these shortcomings, the automatic analysis of cloud cover does seem feasible. A full field trial using a portable system is planned for later this year which will provide an opportunity to collect a large body of classified data for future study and to compare the present system against the judgement of a trained meteorological observer.

Acknowledgements: This work was carried out with support from the Science and Engineering Research Council, under CASE award 90593345, in collaboration with the British Meteorological Office.

\section{References}

[1] J.A. Parikh and A. Rosenfeld, "Automatic Segmentation and Classification of Infrared Meteorological Satellite Data", IEEE Transactions on Systems, Man, and Cybernetics, SMC-8, No.10, 1978., pp 736-743.

[2] K.S. Lau and G. Wade, "Spatial-spectral clustering using recursive spanning trees", IEE Proceedings-I, Vol. 138, No. 4, August 1991, pp

[3] Z.Q. Gu et al, "Comparison of techniques for measuring cloud texture in remotely sensed satellite meteorological image data", IEE Proceedings, Vol. 136, Pt F, No. 5, October 1989, pp 232-238.

[4] C.A. Coomes and A.W. Harrison, "Radiometric Estimation of Cloud Cover", Journal of Atmospheric and Oceanic Technology, Vol. 2, 1984, pp 482-490.

[5] R.J. Schalkoff, "Digital Image Processing and Computer Vision", John Wiley \& sons INC. 1989.

[6] J. Foley, A. van Dam, S. Feiner and J. Hughes, "Computer Graphics: principles and practice", 2nd Edition, Addison-Wesley 1990, 579-585.

[7] A.P. Pentland, "Fractal based description of natural scenes", IEEE Transactions, 1984; PAMI-6, pp 661-674.

[8] T.J. Dennis and N.G. Dessipris, "Fractal modelling in image texture analysis", IEE Proceedings, 1989, Vol. 136, No.5, pp 227-235.

[9] K.I. Laws, "Textured Images Segmentation", $\mathrm{PhD}$ dissertation, University of Southern California, 1980.

[10] M. Unser, "Local linear transforms for texture measurements", Signal Processing 11, 1986, pp. 61-79. 